\title{
An Integrative Approach to Gain Insights into the Cellular Function of Human Ataxin-2
}

\section{Markus Ralser ${ }^{1} \dagger$, Mario Albrecht ${ }^{2 \star} \dagger$, Ute Nonhoff ${ }^{1}$, Thomas Lengauer ${ }^{2}$ Hans Lehrach ${ }^{1}$ and Sylvia Krobitsch ${ }^{1 *}$}

${ }^{1}$ Max Planck Institute for

Molecular Genetics, Ihnestrasse

73, 14195 Berlin, Germany

${ }^{2}$ Max Planck Institute for

Informatics

Stuhlsatzenhausweg 85, 66123

Saarbrücken, Germany
Spinocerebellar ataxia type 2 (SCA2) is a hereditary neurodegenerative disorder caused by a trinucleotide expansion in the SCA2 gene, encoding a polyglutamine stretch in the gene product ataxin-2 (ATX2), whose cellular function is unknown. However, ATX2 interacts with A2BP1, a protein containing an RNA-recognition motif, and the existence of an interaction motif for the C-terminal domain of the poly(A)-binding protein (PABC) as well as an Lsm (Like Sm) domain in ATX2 suggest that ATX2 like its yeast homolog Pbp1 might be involved in RNA metabolism. Here, we show that, similar to Pbp1, ATX2 suppresses the petite (pet ${ }^{-}$) phenotype of $\Delta m r s 2$ yeast strains lacking mitochondrial group II introns. This finding points to a close functional relationship between the two homologs. To gain insight into potential functions of ATX2, we also generated a comprehensive protein interaction network for Pbp1 from publicly available databases, which implicates Pbp1 in diverse RNA-processing pathways. The functional relationship of $\mathrm{ATX} 2$ and $\mathrm{Pbp} 1$ is further corroborated by the experimental confirmation of the predicted interaction of ATX2 with the cytoplasmic poly(A)-binding protein 1 (PABP) using yeast-2-hybrid analysis as well as co-immunoprecipitation experiments. Immunofluorescence studies revealed that ATX2 and PABP co-localize in mammalian cells, remarkably, even under conditions in which PABP accumulates in distinct cytoplasmic foci representing sites of mRNA triage.

(C) 2004 Elsevier Ltd. All rights reserved.

Keywords: polyglutamine disorder; spinocerebellar ataxia type 2; ataxin-2; Pbp1; poly(A)-binding protein leading to ataxia and ultimately death. ${ }^{4}$ Thus, SCA2 belongs to the family of polyglutamine disorders that includes Huntington's disease (HD), dentatorubral-pallidoluysian atrophy (DRPLA), spinal and bulbar muscular atrophy (SBMA) and several other spinocerebellar ataxia types such as SCA1, 3, 6, 7 and $17 .^{4-9}$ The causative gene products of these disorders appear not to be evolutionarily related, and the only common feature is the polyglutamine stretch. Although the various transcripts are widely expressed in brain and many other tissues, the pathogenic observations are restricted to specific neuronal subpopulations. The formation of intranuclear inclusions and alterations of protein interactions due to polyglutamine expansion were shown to be involved in pathogenesis of most polyglutamine disorders. ${ }^{10}$ In contrast, formation of intranuclear inclusions has not been observed in primarily degenerating Purkinje cells in the cerebellum and brainstem of SCA2 patients. ${ }^{11,12}$ Recently, Huynh and colleagues provided 
additional evidence that intranuclear aggregates might not be obligatory for pathogenesis in SCA2. The expression of ATX2 with an expanded polyglutamine stretch resulted in the disruption of Golgi morphology, thus, rather linking cell death and stability of the Golgi complex. ${ }^{13}$

The biological function of ATX2 is not understood to date. A study performed to identify genes associated with cell death in human neuroblastoma cells suggests that ATX2 is involved in regulating the sensitivity to apoptotic stimuli. ${ }^{14}$ The interaction of the related thrombopoietin and erythropoietin receptors TPO-R and EPO-R with an ATX2 homo$\log$, termed A2D, seemed to point to a functional role in cytokine signaling pathways. ${ }^{15}$ Yeast-2hybrid studies identified ATX2 as interaction partner of A2BP1 (ATX2 binding protein 1), a protein of unknown function, but with a putative RNA-recognition motif (RRM domain). ${ }^{16}$ A Caenorhabditis elegans homolog of A2BP1, termed FOX-1, binds RNA and regulates tissue specific alternative splicing. ${ }^{17}$ Remarkably, ATX2 contains an Lsm (Like Sm) domain, ${ }^{18}$ which has been predicted to be capable of RNA binding as in other Lsm domain proteins. ${ }^{19}$ Lsm proteins are generally involved in important processes of RNA metabolism including RNA modification, splicing, and degradation. ${ }^{20}$ Therefore, ATX2 may fulfill similar functions in RNA metabolism. Other studies showed that the C. elegans ATX2 homolog plays a vital role in both embryonic as well as postembryonic development. ${ }^{21,22}$ Moreover, this worm homolog of ATX2 might also be involved in the generation of cellular polarity. ${ }^{21}$ The Drosophila melanogaster ATX2 homolog has been reported to be a dosage-sensitive regulator of cytoskeletal actin filament formation. ${ }^{23}$ Both reduction and overexpression of this homolog in flies cause severe phenotypes such as female sterility, lethality, or degeneration of tissues due to defects in actin filament formation. Since this fly homolog of ATX2 does not directly interact with actin filaments, the effects might result from controlling translation, stability or localization of mRNA encoding proteins involved in actin polymerization. ${ }^{23}$

The yeast homolog of ATX2, named Pbp1 (Pab1binding protein 1, also known as Mrs16), has also been implicated in RNA metabolism and contains an Lsm domain like ATX2 ${ }^{19}$ Originally, Pbp1 has been identified in a suppressor screen for group II intron splicing defects in $\Delta m r s 2$ yeast strains. ${ }^{24}$ $\mathrm{Pbp} 1$ regulates polyadenylation and binds the C-terminal part of the poly(A)-binding protein Pab1, the yeast homolog of human cytoplasmic poly(A)-binding protein 1 (PABP). ${ }^{25,26}$ In the absence of $\mathrm{Pbp} 1$, the $3^{\prime}$-ends of pre-mRNAs are cleaved properly, but lack a complete poly(A) tail. ${ }^{25}$ A recent study has identified the poly(A)-polymerase-interacting proteins Fir1 and Ufd1, which have been implicated in mRNA $3^{\prime}$-end processing, and the transcription factor Dig1, as novel interaction partners of $\mathrm{Pbp} 1 .{ }^{27}$ In addition, $\mathrm{Pbp} 1$ negatively regulates the activity of the poly(A)-nuclease (PAN) by interacting with the subunit Pan2 of the PAN complex Pan2-Pan3. ${ }^{28}$

Interestingly, another recent study showed that loss of Pbp1 expression causes reduced expression of the $\mathrm{HO}$ endonuclease at the posttranscriptional level, and that a complex of Pbp1 and the putative nuclease Mkt1 regulates the translation of the $\mathrm{HO}$ endonuclease gene via the $3^{\prime}$ untranslated region (UTR) of $\mathrm{HO}$ mRNA. ${ }^{29}$

In this report, we will shed new light on related functions of ATX2 and its yeast homolog Pbp1, and provide data suggesting that ATX2 may function in RNA metabolism.

\section{Results}

\section{Human ATX2 and yeast Pbp1 are functionally related}

Even though ATX2 and Pbp1 possess weak sequence similarity in regions that are outside globular domains and predicted to be mainly flexible and intrinsically unstructured, both proteins have very similar protein architectures (Figure 1). They consist of a putative RNA-binding Lsm domain followed by another as yet uncharacterized Lsm-associated domain LsmAD and a C-terminal PABP/Pab1-binding region. ${ }^{19,30,31}$ In order to gain insight into cellular functions of ATX2, we investigated whether ATX2 and Pbp1 might be functionally related. Initially, Pbp1 was identified in a genetic screen performed to isolate suppressors of mitochondrial group II intronsplicing defects in $\Delta m r s 2$ yeast strains. ${ }^{24}$ The deletion of MRS2, whose gene product is essential for intron splicing, $\mathrm{Mg}^{2+}$ transport and homeostasis in yeast mitochondria, leads to a respiratorydefective petite phenotype (pet ${ }^{-}$phenotype) indicated by failure of $\Delta m r s 2$ strains to grow on glycerol as carbon source. Deletion of mitochondrial group II introns in $\Delta m r s 2$ strains reduces the severity of the pet $^{-}$phenotype. In this strain background, the pet ${ }^{-}$ phenotype is suppressed by overexpression of Pbp1. Additionally, suppression of the pet ${ }^{-}$ phenotype also occurs in strains deleted for group I and group II introns. ${ }^{24,32,33}$

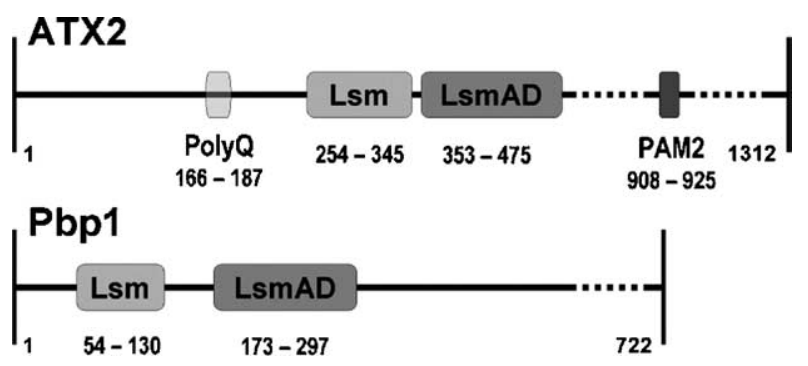

Figure 1. Domain architecture of human ATX2 and its yeast homolog $\mathrm{Pbp} 1$. Both proteins have a putative RNAbinding Lsm domain followed by an as yet uncharacterized Lsm-associated domain LsmAD. ATX2 also contains a PABC-interacting motif named PAM2. 
To determine whether ATX2 is capable of suppressing the pet $^{-}$phenotype like yeast $\mathrm{Pbp} 1$ does, we transformed various isogenic $\Delta m r s 2$ strains with different mitochondrial intron content as well as the corresponding wild-type yeast strains with the low-copy plasmid p416-ATX2 for expression of ATX2 or empty vector as control. Hereinafter, transformants were grown in liquid selective medium, and yeast cultures were transferred onto glycerol medium supplemented with $0.15 \%$ glucose as well as onto glucose plates as control. No growth difference was observed between the respective MRS2 wild-type or $\Delta m r s 2$ strains containing either empty vector or expressing ATX2 on glucose plates (data not shown). However, on glycerol plates, $\Delta m r s 2$ yeast strains containing the empty vector showed the expected pet phenotype that was not observed among MRS2 strains (Figure 2). Strikingly, yeast colonies without mitochondrial group II introns as well as without mitochondrial group I and II introns exhibited significantly improved growth when expressing ATX2 (Figure 2). As described for Pbp1, no suppression occurred in the $\Delta m r s 2$ strain DBY747 WT containing all introns. Thus, ATX2 partially suppresses the observed pet ${ }^{-}$phenotype of the respective $\Delta m r s 2$ yeast strains. We also performed this experiment with ATX2 containing an expanded polyglutamine tract of 79 glutamines, and observed the same results (data not shown).

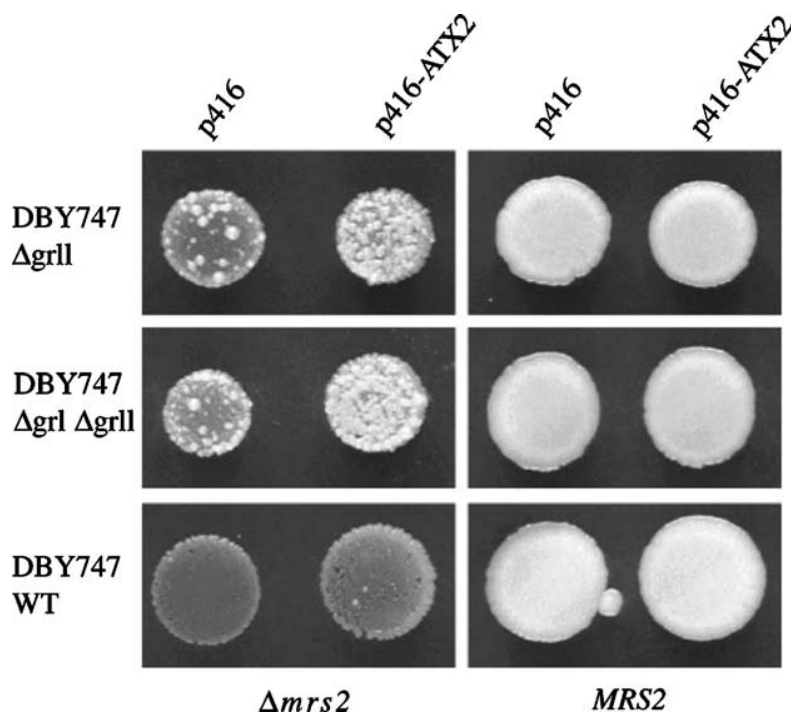

Figure 2. ATX2 suppresses the pet ${ }^{-}$phenotype of $\Delta m r s 2$ yeast strains without mitochondrial group II introns as well as without mitochondrial group I and II introns. $\Delta m r s 2$ yeast strains DBY747 $\Delta$ grII (without mitochondrial group II introns), DBY747 $\operatorname{grI} \Delta$ grII (without mitochondrial group I and II introns), and DBY747 WT (with mitochondrial introns) along with the corresponding

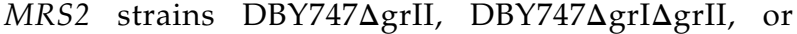
DBY747 WT as controls were transformed with plasmid p416GPD or p416-ATX2. Afterwards, transformants were spotted onto SD glycerol medium lacking uracil, and plates were incubated for seven days at $30^{\circ} \mathrm{C}$.
Therefore, our data suggest that ATX2 and its yeast homolog $\mathrm{Pbp} 1$ possess functional similarities.

\section{Analysis of Pbp1 functions}

Since ATX2 and Pbp1 seem to share functional similarities, we exploited publicly available protein interaction data assembled in the GRID database to draw a yeast interaction network for Pbp1 (Figure 3 and Supplementary Figure A) in order to learn more about potential functions of ATX2. Although the reliability of such interaction maps based on high-throughput experiments must be regarded with some caution, the map can be used to gain a global perspective on Pbp1 function. The map indicates that Pbp1 binds the DEAD/H-box RNAhelicase Dhh1 that plays a major role in mRNA turnover and degradation. ${ }^{34-36}$ Furthermore, Pbp1 is clearly linked to the nuclear export machinery of $\mathrm{mRNA}^{37}$ by the exportin $\mathrm{Crm}^{38}$ and the nucleoporin Nup145. ${ }^{39}$ These findings match experimental results that observed Crm1 in complex with other nucleoporins and RanGTP. ${ }^{40} \mathrm{Pbp} 1$ is also associated with the subunit $h$ of the ATP synthase Atp $14^{41}$ and the tRNA-specific adenosine

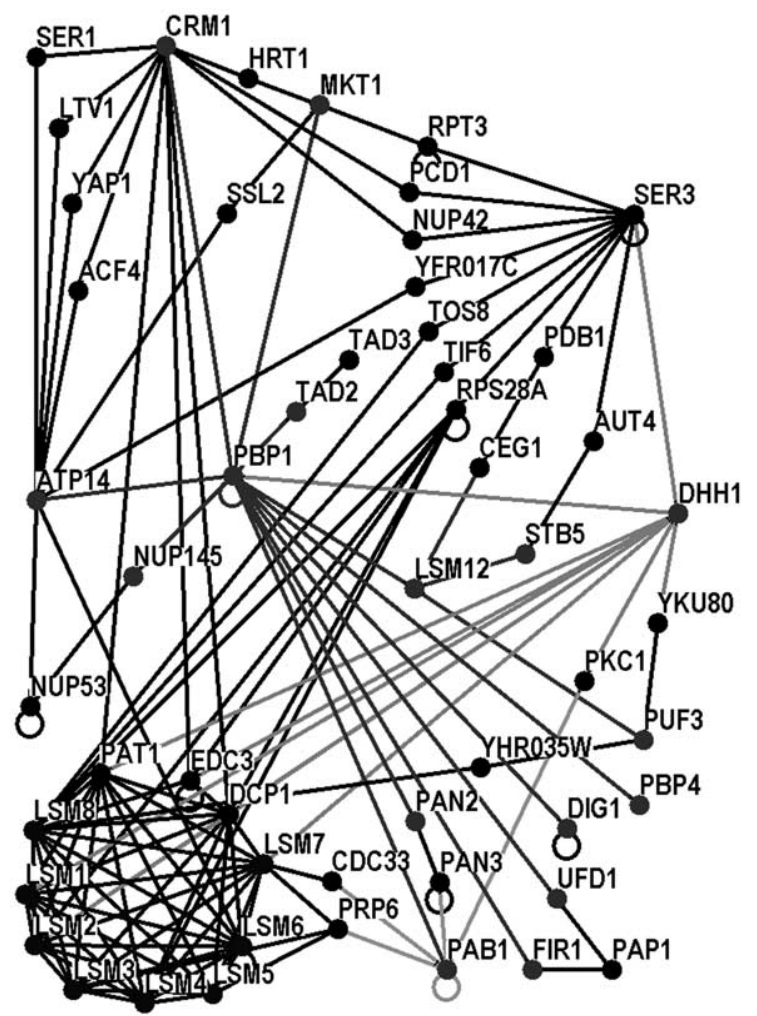

Figure 3. Yeast protein interaction map with focus on $\mathrm{Pbp} 1$ and Lsm12 (Supplementary Figure A provides more details). Each node represents a different Saccharomyces cerevisiae protein, whose association with another protein is indicated by an edge. The dense interaction network of the Lsm1-8 proteins is arranged in a circle. Edges emanating from the ATX2 homolog Ppb1 or the RNAhelicase Dhh1 are colored respectively in dark or light gray. 
deaminase subunit Tad2/ADAT2, ${ }^{42}$ which belongs to a conserved eukaryotic family of RNA-dependent deaminases including ADARs that edit doublestranded pre-mRNA to convert adenosine to inosine, presumably in order to fine-tune biological pathways. $^{43}$ At least human ADAR1 contains a functional N-terminal nuclear export signal and forms a tripartite export complex with CRM1 and RanGAP. ${ }^{44}$

We also included the recently described interactions of Pbp1 with Mkt1, Pan2, Fir1, Ufd1, Dig1, and the as yet uncharacterized protein $\mathrm{Pbp} 4$ in the network. ${ }^{27-29}$ We observed that the putative nuclease Mkt1 and the poly(A)-nuclease subunit Pan2 not only interact directly with $\mathrm{Pbp} 1$, but are also linked to $\mathrm{Pbp} 1$ indirectly via other interaction partners. This may point to larger protein complexes containing Pbp1 and Mkt1 or Pan3 and other proteins. Fir1 and Ufd1 have been implicated in mRNA 3 -end processing and interact with the poly(A)-polymerase. ${ }^{45,46}$ Fir1 also binds the processing factor Ref2, which presumably links the cleavage and polyadenylation factor CPF to the cleavage factor subunit CFIA. ${ }^{45}$ Interestingly, Ufd1 interacts with $\mathrm{Uba} 2$, and both proteins are shown to be associated with the poly(A)-polymerase and are contributing to its activity. ${ }^{46}$ Dig1 associates with the mitogen-activated protein kinase $\mathrm{MAPK}^{47}$ and is important for transcriptional repression of mating-type specific gene sets. ${ }^{48}$ Furthermore, $\mathrm{Pbp} 1$ has been shown experimentally to interact with the poly(A)-binding protein Pab1. ${ }^{25}$ In general, the C-terminal region of Pab1 has been determined to act as a protein-protein interaction domain recruiting translation factors or other cellular factors necessary for the organization of the mRNA ribonucleic acid complex. ${ }^{26}$

Remarkably, a novel interaction partner of $\mathrm{Pbp} 1$ is the protein Yhr121w of as yet unknown function, but this protein was recently named Lsm12 based on the structure prediction of an N-terminal Lsm domain. ${ }^{31}$ This Lsm domain is followed by a long C-terminal tail, which may contain another RNAbinding domain involved in tRNA splicing. ${ }^{31}$ Lsm12 associates with the transcription factor Stb5, which interacts with the transcriptional repressor $\operatorname{Sin} 3{ }^{49}$ the mRNA guanylyltransferase Ceg1 of an mRNA capping enzyme complex including the triphosphatase Cet $1,{ }^{50}$ and the RNAbinding protein Puf3. Puf3 is a member of the family of pumilio-like Puf repeat containing proteins ${ }^{51}$ and functions as transcript-specific regulator of mRNA degradation. ${ }^{52}$ Puf3 has been suspected as a repressor involved in the movement of mRNA to $\mathrm{P}$ bodies. ${ }^{53}$ In summary, Pbp1 is involved in important RNA-processing pathways such as RNA editing, pre-mRNA splicing, mRNA export and degradation.

\section{ATX2 interacts with the C-terminal domain of PABP}

To investigate whether the yeast data can be assigned to human ATX2, we focused on the central interaction of $\mathrm{Pbp} 1$ with Pab1 observed in yeast. In particular, the C-terminal region of human ATX2 has been predicted to contain a PABP-interacting motif termed PAM2 (amino acid residues 908-925) for the C-terminal PABC domain of PABP. ${ }^{30,54}$ It has also been reported that the $C$. elegans ATX2 homolog is contained in a complex together with C. elegans PAB1. ${ }^{22}$ However, a direct interaction between the C. elegans ATX2 homolog and PAB1 has not been demonstrated in this study. ${ }^{22}$ Therefore, we carried out a yeast-2-hybrid analysis to investigate in an experimental system whether human ATX2 directly interacts with the human PABC domain. We observed that yeast expressing the fusion proteins lexA-ATX2 $816-1312$ and AD-PABP ${ }_{498-635}$ was able to grow on SD medium lacking uracil and histidine indicating the activity of the reporter genes URA3 and HIS3. The activity of the LacZ reporter gene was observed by a blue color shift in the $\beta$-galactosidase assay (Figure $4 \mathrm{~A}$ ). To determine that this interaction is specific, yeast cells expressing proteins lexA-ATX2 $816-1312$ and AD, LexA and ADPABP $_{498-635}$, or lexA-httQ20 and AD-PABP $498-635$ were also spotted onto the appropriate SD medium plates or on membrane for the $\beta$-galactosidase assay. In addition, the relative activity of the $L a c Z$ reporter gene product was measured by a liquid $\beta$-galactosidase assay as described in Materials and Methods (Figure 4B). No activity of the three reporter genes was observed in controls, demonstrating that the interaction of ATX2 and PABC is specific.

In a next step, we investigated whether the interaction of ATX2 and PABC also occurs in mammalian cell lines. First, we transiently transfected COS-1 cells with plasmids encoding the fusion proteins Flag-PABP $498-635$ and HAATX $2_{816-1312}$ or with the respective control plasmids. After 48 hours, total cell lysates were prepared and immunoprecipitation was performed with an antibody directed against the HA-tag. The resulting samples were analyzed by SDS-PAGE and immunoblotted using an antibody directed against the FLAG-tag (Figure 4C). This experiment showed that PABC was precipitated with an antibody directed against the HA-tag (Figure 4C, lower panel, lane 4). In control lysates, no PABC protein was precipitated (Figure 4C, lower panel, lanes 1-3). Second, we performed immunofluorescence microscopy and showed that endogenous PABP and ATX2 are present in the cytoplasm of COS-1 cells, and discovered that both proteins do co-localize in certain areas of the cytoplasm (Figure 4D). These results indicate that an interaction of ATX2 and PABP occurs in mammalian cells. Noticeably, it seems that ATX2 and Pbp1 use different mechanisms to interact with the C-terminal part of PABP and Pab1, respectively. While ATX2 binds the PABC domain itself, the major interaction site of $\mathrm{Pbp} 1$ with $\mathrm{Pab} 1$ appears to be a region preceding the PABC domain of Pab1. ${ }^{28}$ The observed different binding modes of ATX2 and 
(A)

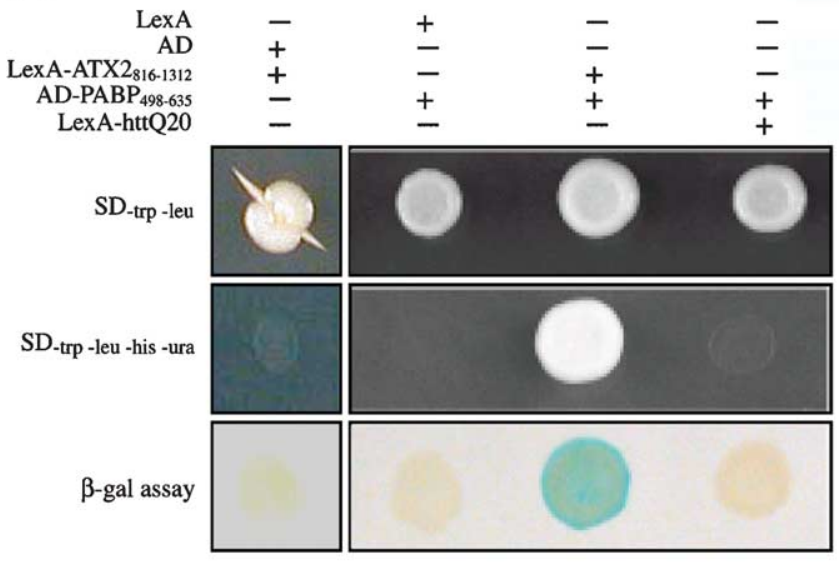

(C)

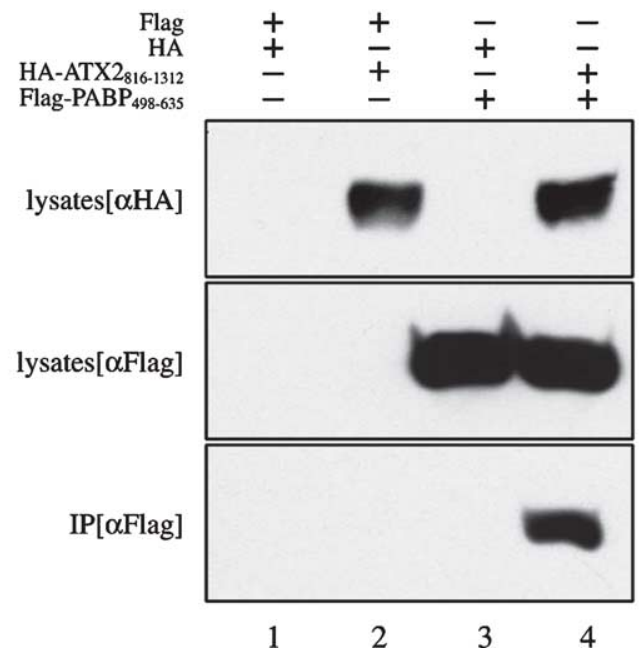

(B)

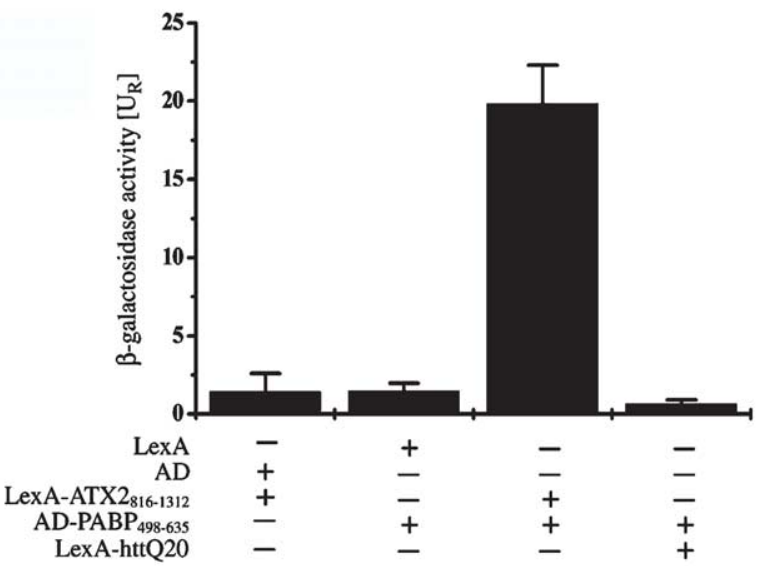

(D)
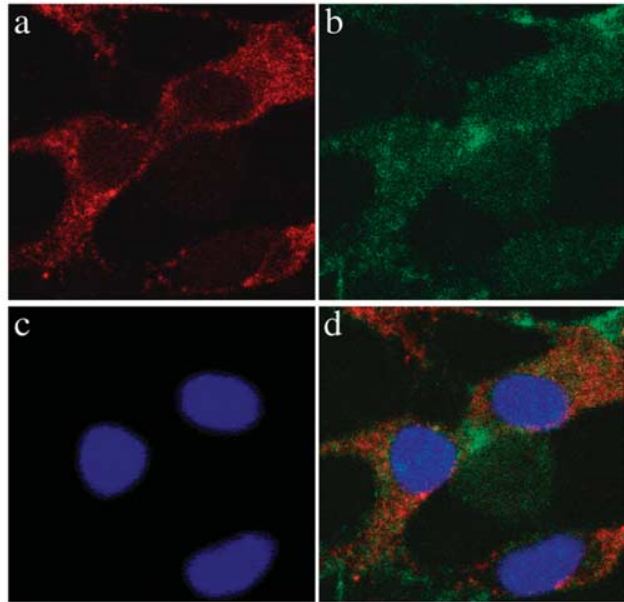

Figure 4. ATX2 interacts with PABC. A, Yeast expressing proteins lexA-ATX2 $816-1312$ and AD, LexA and ADPABP $_{498-635}$, lexA-ATX2 $2_{816-1312}$ and AD-PABP ${ }_{498-635}$ or AD-PABP ${ }_{498-635}$ and lexA-httQ20 were spotted onto selective media lacking the respective amino acids or on membrane for detection of $\beta$-galactosidase activity. Plates were incubated for three to five days at $30{ }^{\circ} \mathrm{C}$, growth of yeast was analyzed, and $\beta$-galactosidase activity was determined. $B$, Liquid $\beta$-galactosidase assay. The relative activity of $\beta$-galactosidase was measured as described in Materials and Methods. Each column represents the mean value of four measurements. Error bars indicate the standard deviation. C, Co-immunoprecipitation of ATX2 and PABC. COS-1 cells were transiently transfected with the plasmids pTL1-HAATX2-FD and pTL-FLAG-PABC, or with control plasmids. SDS-PAGE and Western blotting were performed to visualize the expression of tagged proteins (upper and middle panel). Total cell lysates were precipitated with antibody directed against HA-tag, and precipitates were visualized with an antibody directed against FLAG-tag (lower panel). D, Colocalization of ATX2 and PABP. COS-1 cells were stained with antibodies directed against (a) PABP and (b) ATX2. (c) DAPI-stained nuclei. (d) Merged confocal image of $a, b$ and c.

Pbp1 to PABC point to common evolutionary changes of the protein binding interface and are consistent with the fact that ATX2 contains the PABC-interacting motif PAM2, but not Pbp1. In agreement with that, we could not observe an interaction of human ATX2 and the corresponding region of the yeast PABP homolog Pab1 in the yeast2-hybrid system (data not shown).

\section{ATX2 is a component of mammalian stress granules}

Stress granules (SGs) are cytoplasmic sites into which untranslated mRNAs are stalled during environmental stresses, and both RNA stabilizing or destabilizing proteins are recruited to SGs. ${ }^{55}$ Given that PABP is one of the components and interacts with ATX2 in transiently transfected mammalian cell lines, we investigated whether ATX2 is also a component of SGs. Since the formation of SGs is induced by various stresses, for example, heat shock, osmotic shock or oxidative stress, we performed co-localization studies using COS-1 cells that had been heat-treated for the induction of SGs. Immuno-staining of endogenous PABP was performed to visualize SGs (Figure 5A). Remarkably, ATX2 formed distinct foci (Figure 5B) that co-localized with PABP 

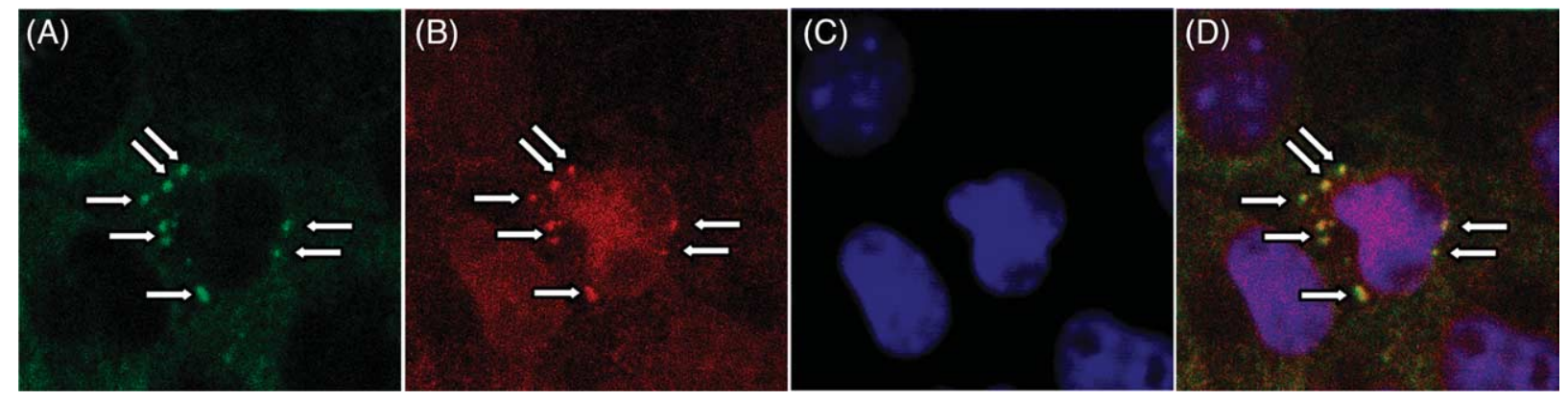

Figure 5. ATX2 and PABP co-localize in stress granules. Localization of endogenous ATX2 and PABP in heat-treated COS-1 has been analyzed by immunofluorescence microscopy. SGs were visualized by staining of (A) PABP and (B) ATX2. (C) DAPI-stained nuclei. (D) Merged confocal image of (A), (B) and (C). Arrows indicate SGs. In this experiment, SCA2B antibody was diluted $1: 200$. PABP was visualized with a FITC-labeled secondary antibody and ATX2 with a CY3-labeled secondary antibody. The image chosen displays a representative cell.

(Figure 5D). Thus, these data demonstrated that ATX2 is part of SGs in heat-stressed cells.

\section{Discussion}

The molecular function of the $S C A 2$ gene product ATX2 has not been understood to date. However, some evidence provided by studying ATX2 homologs from yeast, $C$. elegans, D. melanogaster, and man indicates that they are implicated in RNA-processing pathways. ${ }^{16,21,23,25,29}$ In this study, we present further data pointing to a potential role of ATX2 in RNA metabolism. We discovered a close functional relationship between ATX2 and its yeast homolog $\mathrm{Pbp} 1$. Firstly, $\mathrm{Pbp} 1$ is a suppressor of the pet phenotype of $\Delta m r s 2$ yeast strains lacking mitochondrial group II or group I and II introns, ${ }^{24}$ and we showed that ATX2 suppresses this pet ${ }^{-}$phenotype of the respective $\Delta m r s 2$ yeast strains like its yeast counterpart.

The yeast interaction map for $\mathrm{Pbp} 1$ revealed that this protein appears to be involved in essential RNA-processing tasks. The RNA-helicase Dhh1 binds many Lsm domain proteins and forms part of complexes involved in mRNA decapping, deadenylation and subsequent decay. ${ }^{34-36}$ In addition, the association of Pbp1 and Lsm12 suggests that the Lsm domains of both proteins may assemble as heteromers as it is known from many other Lsm domain proteins. ${ }^{20}$ Taken the length of about 722 and 1312 amino acid residues, respectively, into account, Pbp1 and ATX2 may form part of large complexes and work as scaffolding proteins.

This hypothesis is supported by the observation that Lsm12 also associates with different proteins functioning in mRNA metabolism. For instance, Lsm12 associates with Puf3, which regulates mRNA degradation by binding the $3^{\prime}$ UTR of target mRNA and enhancing its deadenylation rate. ${ }^{52}$ In addition, the Pbp1 interaction partner Mkt1 also regulates mRNA translation via the $3^{\prime}$ UTR, and thus Pbp1 may mediate mRNA modification by nuclease activity of Mkt1. ${ }^{29}$ Furthermore, the
poly(A)-nuclease subunit Pan2 binds to $\mathrm{Pbp} 1,{ }^{27}$ and the poly(A)-polymerase binding proteins Fir1 and Ufd1 interact with Pbp1 and have also been implicated in processing the $3^{\prime}$-end of mRNA. ${ }^{28}$

Since a functional relationship of ATX2 and Pbp1 has been corroborated by our yeast suppression studies, it is worthwhile to reason that ATX2 might also bind nucleic acids and RNA-modifying proteins that are human equivalents or even homologs of the corresponding yeast $\mathrm{Pbp} 1$-interacting proteins. We confirmed the interaction of ATX2 with the PABC domain of PABP by yeast-2-hybrid studies and co-immunoprecipitation experiments using mammalian cells. This interaction links ATX2 to RNA metabolism, since PABP, which is found complexed to the $3^{\prime}$-end of poly(A) tails of eukaryotic mRNA, is required for poly(A) shortening and translation initiation. ${ }^{56}$ It mediates mRNA circularization through binding of the eIF4F translation initiation complex, which enhances translation by facilitating the recycling of ribosomes. ${ }^{26}$ PABP consists of four N-terminal RNA-recognition motifs (RRMs) and a C-terminal domain termed PABC that has been shown to be important for functional regulation by interacting with many cytosolic and nuclear proteins. ${ }^{30}$

In addition, PABP is found in distinct cytoplasmic granules arising in the cytoplasm of mammalian cells in response to environmental stresses that have been termed stress granules. ${ }^{55,57}$ SGs appear to be in a dynamic equilibrium with polysomes and assemble and disassemble very rapidly in the cytoplasm. They represent sites where mRNA stability and translability is regulated, and they contain several ribonucleoproteins as well as a number of mRNA stabilizing and destabilizing proteins. ${ }^{57}$ Since we discovered that ATX2 is a component of these sites of mRNA triage, one might speculate that ATX2 plays a role in regulating cellular mRNA turnover. Along this line, it has been suggested that the D. melanogaster homolog of ATX2 might control different RNAs encoding actin mediators. ${ }^{23}$ Furthermore, the C. elegans ATX2 homolog occurs in complexes together with 
C. elegans PAB1 and may be implicated in translational regulation. ${ }^{22}$

The first evidence that ATX2 might be involved in RNA metabolism was provided by Shibata and colleagues. ${ }^{16}$ They identified A2BP1, which is also known as HRNBP1 (hexaribonucleotide binding protein 1) and contains one RNA-recognition motif (RRM domain), as an interaction partner of ATX2. To further elucidate the potential functions of A2BP1, we assembled a multiple sequence alignment of eukaryotic homologs that are closely related to A2BP1 and also contain a single RRM domain (Supplementary Figure B). This alignment does not contain a protein from yeast because the distant evolutionary relationship does not allow for the clear identification of a putative functional homolog of A2BP1. Several yeast proteins with only one RRM domain are listed in the Pfam domain database, but none of them has been reported to interact with $\mathrm{Pbp}$.

The human A2BP1 paralog HRNBP2/ FXH/RTA/TH82 (hexaribonucleotide binding protein 2/FOX1 homolog/repressor of tamoxifen (an anti-estrogen) transcriptional activity/Fynbinding molecule $\mathrm{TH}$ 82) has been observed to localize to nuclear speckles, to bind RNA and to interact with the Src family tyrosine kinase Fyn. ${ }^{58,59}$ HRNBP2 also works as a negative co-regulator for the transcriptional activity of the estrogen receptor ER $\alpha$. The expression of HRNBP2 is induced by androgen stimulation. ${ }^{60}$ One C. elegans homolog of $\mathrm{A} 2 \mathrm{BP} 1$ has been named FOX-1, which posttranscriptionally regulates as numerator element the expression of the master sex-determination switch XOL-1, ${ }^{61-64}$ binds RNA and regulates tissuespecific alternative pre-mRNA splicing. ${ }^{17}$

Interestingly, another C. elegans A2BP1/FOX-1 homolog/paralog is SPN-4/GEI-20 (spindle-orientation defective protein 4/GEX-3 interacting protein 20). This protein plays diverse roles in the translational control of cell-fate specification and patterning the early C. elegans embryo ${ }^{65-69}$ which is consistent with the observed essential role of the C. elegans homolog of ATX2 in embryonic development. ${ }^{21}$ Similar to the Lsm12 interacting yeast protein Puf3, whose close yeast homologs Puf1/2 possess one RRM domain, SPN-4 binds the $3^{\prime}$ UTR of mRNA. It specifically interacts with a subregion of this UTR, which contains a temporal and spatial inhibition and activation region of translation, respectively. Generally, some mRNAs possess a cytoplasmic polyadenylation element (CPE) in the $3^{\prime}$ UTR, which is bound by CPE-binding proteins (CPEB) and involved in changing the length of the poly(A) tail. An elongation of this tail is assumed to lead to translational activation. Therefore, SNP-4 may be part of large complexes and also interacts with several other RNA-binding proteins such as GEX-3, MEX-3, and POS-1 implicated in RNA binding, transcriptional and translational regulation of embryogenesis, tissue morphogenesis, and cell polarity and migration. ${ }^{22,65-70}$ Moreover, GEX-3 forms a complex with GEX-2 at cell boundaries in embryos, whose closely related human and fly proteins NAP1 (Nck-associated protein 1) and Kette interact with the Rho-GTPase Rac1 and are required for axonal development and appropriate actin organization like the ATX2 homolog of D. melanogaster. $23,70,71$

Overall, a bulk of evidence exists that ATX2 homologs might be involved in RNA splicing, mRNA stabilization or degradation and other essential RNA-processing pathways. Evidently, further studies are required to define the function of ATX2 in RNA metabolism. It also remains to be demonstrated in experiment which of the numerous human ATX2 homologs, ataxin-2 itself or one of its close human paralogs named ataxin-2-like and related proteins, is the true functional ortholog of yeast Pbp1 and C. elegans ATX2. In the future, we will focus on investigating further protein interactions that have been predicted from the yeast $\mathrm{Pbp} 1$ interaction network, since most of the identified $\mathrm{Pbp} 1$ interaction partners have human homologs. Two important candidates are the as yet uncharacterized Lsm domain protein Lsm12 (human FLJ30656) and the evolutionarily and functionally conserved RNA-helicase Dhh1 (human p54/RCK), which is known to interact with many other Lsm proteins to unwind RNA. ${ }^{36}$ Elucidation of the pathways, in which ATX-2 is involved, could contribute to identify the pathomechanisms underlying SCA2.

Strikingly, disturbances in RNA-processing pathways seem to underlie pathogenesis of the polyglutamine disorder SCA1. Ataxin-1 (ATX1) contains an $\mathrm{AXH}$ domain with an $\mathrm{OB}$ fold for binding RNA. $^{72,73}$ Alterations in RNA metabolism have been suggested to be involved in pathogenesis of SCA1, since binding of ATX1 to RNA lessens in a polyglutamine-dependent manner. ${ }^{74}$ Furthermore, several RNA-binding proteins have been identified in a screen to modify ATX1-induced neurodegeneration. ${ }^{75}$ Altogether, changes in RNA metabolism might be a common feature contributing to the pathogenesis of some polyglutamine disorders, and the exploration of protein interaction networks for related proteins in other organisms may aid in the elucidation of the molecular disease processes.

\section{Materials and Methods}

\section{Plasmids}

To generate plasmids for expression of ATX2 in yeast and mammalian cells, we carried out the following cloning strategies.

For generation of yeast-2-hybrid expression constructs, a DNA fragment encoding amino acids 816-1312 of ATX2 was amplified by PCR from plasmid pcDNA1.1-Sca2Q22 with primer pair a2-s-3 (5' CG GAA TTC CTA CTA AAC AAA AAT AGA GAG $3^{\prime}$ ), introducing an EcoRI site, and a2-as-3 (5'GCG GTC GAC TTA CAA CTG CTG TTG 3'), introducing a Sall site. The resultant PCR product was treated with EcoRI and SalI, and subcloned into the 
EcoRI/SalI sites of vector pBTM116 to create plasmid pBTM-ATX2-FD. For plasmid pGAD-PABC, a DNA fragment encoding amino acids 498-635 matching the PABC domain of PABP $^{30}$ was amplified via PCR with primer pair PABC-s-1 (5' GAG TCG ACA GCC GCT GCA GCT ACT CCT $3^{\prime}$ ), incorporating an Sall site, and PABCas-1 (5' GAT CGC GGC CGC TTA AAC AGT TGG AAC ACC $3^{\prime}$ ), incorporating an NotI site, from a human fetal brain cDNA-library (Clontech). The resulting SalI/NotI fragment was inserted into the respective sites of vector pGAD426. The plasmid pBTM-CAG20 is described elsewhere. $^{76}$

Plasmid pTL1-HA-ATX2-FD was obtained by subcloning an EcoRI/KpnI fragment isolated from pBTMATX2-FD into the corresponding sites of vector pTL1-HA. Inserting a SalI/NotI fragment from pGAD-PABC into the XhoI/NotI sites of vector pTL-Flag generated plasmid pTL-FLAG-PABC.

To create yeast expression constructs for our suppressor studies, we used plasmid pTL1-HA-ATX2. An N-terminal fragment of the SCA2 coding sequence comprising nucleotides 1-1186 was amplified with primer pair a2-s-1 (5'ATG CGC TCA GCG GCC GCA GC $3^{\prime}$ ) and a2-BamHI-as (5'CAT TGG GAT CCC ATC CAT TAG ATA CGT C $3^{\prime}$ ) from plasmid pcDNA1.1-Sca2Q22. The PCR product was subcloned into vector p426GPD to obtain plasmid p426-ATX2-NT. Subsequent sequence analysis revealed an insertion of one nucleotide close to the $5^{\prime}$-end of the DNA fragment. In a next step, we isolated a $0.98 \mathrm{~kb}$ SacI/BamHI fragment from plasmid p426-ATX2-NT, and amplified just a $0.2 \mathrm{~kb}$ fragment from the $S C A 2 \mathrm{cDNA}$ with primer pair a2-s-2 (5 $5^{\prime}$ GT ATT CTA GAA ACT ATG CGC TCA GCG GCC GC $3^{\prime}$ ) and a2-SacI-as ( $5^{\prime}$ GGA GGG AGG CGA GCT CTG CCG G $3^{\prime}$ ). Both the $0.2 \mathrm{~kb}$ XbaI/SacI fragment and the $0.98 \mathrm{~kb}$ SacI/BamHI fragments were ligated for further cloning purposes into the respective sites of pUC19 to generate pUC19-ATX2-NT. Afterwards, plasmid pUC19-ATX2-NT was digested with XbaI, subsequently treated with Klenow enzyme to blunt ends, and digested with BamHI. In addition, a BamHINotI fragment from plasmid pcDNA1.1-Sca2Q22 was isolated. Both fragments were subcloned into the blunted EcoRI site and NotI site of vector pTL1-HA to create pTL1-HA-ATX2.

Plasmid p416-ATX2 was generated by treating pTL1HA-ATX2 with KpnI, blunting the ends, and subsequent digest with XbaI. The resultant fragment was isolated and subcloned into the blunted HindIII site and XbaI site of p416GPD.

Underlined primer sequences indicate restriction sites. All DNA fragments generated by PCR were verified by sequencing.

In addition, we want to mention that the coding sequence of human SCA2 that was used contains a polymorphism, since an insertion of six nucleotides (GGT AAA) after nucleotide position 2997 was identified via sequence analysis. Publicly available EST sequence data indicated that, in fact, this variation is common, because this polymorphism accounts for approximately half of the available EST sequences (data not shown). The sequence data of this polymorphism was submitted to NCBI dbSNP and accession number ss23142229 was assigned.

\section{Yeast cultivation and transformation}

Yeast strains used in this study are listed in Table 1. Yeast was grown in rich medium (YPD) containing $2 \%$ glucose or minimal medium (SD) deficient for the respective amino acids and supplemented with $2 \%$ glucose or $3 \%$ glycerol $/ 0.15 \%$ glucose. Transformation of yeast strains was performed using the lithium acetate method. $^{77}$

\section{Yeast-2-hybrid analysis}

Yeast strain L40ccua was co-transformed with vector pBTM-ATX2-FD and pGAD, pBTM and pGAD-PABC, pBTM-ATX2-FD and pGAD-PABC, or with pGAD-PABC and pBTM-CAG20, respectively. Transformants were plated onto SD media lacking amino acids tryptophan and leucine. After incubation of plates for three days at $30{ }^{\circ} \mathrm{C}$, single yeast colonies were isolated and tested for the activity of the three reporter genes URA3, HIS3, and $\mathrm{LacZ}$. Therefore, yeast cells were grown in the respective liquid media and spotted onto solid medium lacking leucine and tryptophan, onto solid medium lacking amino acids tryptophan, leucine, uracil and histidine, as well as onto nylon membrane (MSI) for testing activity of the $L a c Z$ reporter gene. Plates were incubated for $3-5$ days at $30^{\circ} \mathrm{C}$. Afterwards, growth on plates was analyzed. For testing activity of the $\mathrm{LacZ}$ reporter gene, the membrane was incubated in liquid nitrogen for lysis of yeast. Subsequently, the membrane was placed on Whatman paper saturated with X-Gal buffer (phosphate buffer $\mathrm{pH} 7.0,0.15 \% \mathrm{X}$-Gal, $10 \mathrm{mM}$ DTT) for up to four to six hours at $37^{\circ} \mathrm{C}$. In addition, the relative activity of the $\mathrm{LacZ}$ gene product was measured by a liquid $\beta$-galactosidase assay. Briefly, yeast cultures grown in logarithmic phase were shock-frozen and resuspended in buffer $\mathrm{H}(100 \mathrm{mM}$ Hepes/KOH, $150 \mathrm{mM} \mathrm{NaCl}, 2 \mathrm{mM}$ $\left.\mathrm{MgCl}_{2}, 1 \% \mathrm{BSA}, \mathrm{pH} 7.0\right)$. After addition of chloroform and SDS to a final concentration of $7 \%$ and $0.007 \%$, respectively, the lysates were intensively mixed. ONPG (o-nitrophenyl- $\beta$-D-galactopyranoside $(1.8 \mu \mathrm{mol} / \mathrm{ml})$, PeqLAB) was added and samples were incubated at $37^{\circ} \mathrm{C}$. The relative $\beta$-galactosidase activity was calculated by formula $U_{\mathrm{R}}=\left(1000 \times \mathrm{OD}_{420}\right) /\left(V_{[\mathrm{ml}]} \times T_{[\mathrm{h}]} \times \mathrm{OD}_{600}\right)$.

\section{Cell cultivation and transfection}

COS-1 cells were cultivated in Dulbecco's modified Eagle medium (Gibco) supplemented with 100 units $/ \mathrm{ml}$ Penicillin/G-Streptomycin (Gibco) and 5\% heatinactivated foetal bovine serum (Biochrom) at $37^{\circ} \mathrm{C}$ and $5 \% \mathrm{CO}_{2}$.

For transient transfections, confluent cells were detached, transferred into six-well plates, and incubated overnight. Co-transfection was performed at a confluence of $60-80 \%$ using the reagent Polyfect (Qiagen) and $1 \mu \mathrm{g}$ of each corresponding plasmid.

\section{Co-immunoprecipitation}

COS- 1 cells were transiently transfected with respective ATX2 and PABC plasmids and incubated for 48 hours at $37^{\circ} \mathrm{C}$ to allow expression of proteins. Afterwards, cells were washed with PBS and lysed in buffer $(50 \mathrm{mM}$ Tris$\mathrm{HCl}, 100 \mathrm{mM} \mathrm{NaCl}, 5 \mathrm{mM} \mathrm{MgCl} 2,0.5 \% \mathrm{NP} 40,1 \mathrm{mM}$ EDTA, $\mathrm{pH} 8.8,250$ units $/ \mathrm{ml}$ benzonase (MERCK) and 2.5\% protease inhibitors ("complete" tablets, Roche)) for 30 minutes on ice. Protein concentration of lysates was determined by Bio-Rad protein assay. $130 \mu \mathrm{g}$ of each cell lysate was incubated with $1 \mu \mathrm{l}$ of monoclonal mouseanti-HA antibody (Roche) for two hours at $4{ }^{\circ} \mathrm{C}$. Afterwards, $15 \mu \mathrm{l}$ sheep anti-mouse IgG-conjugated Dynabeads M-280 (Dynal) were added to each lysate, and samples were incubated for three hours at $4{ }^{\circ} \mathrm{C}$. Dynabeads were pulled down magnetically and beads were washed three 
Table 1. Yeast strains

\begin{tabular}{|c|c|c|c|}
\hline Name & Genotype nuclear & $\begin{array}{l}\text { Genotype } \\
\text { mitochondrial }\end{array}$ & Source \\
\hline DBY747 & MATa leu2-3 leu2-112 his3-1 ura3-52 trp1-289 & rho $^{+}$mit $^{+}$ & $\begin{array}{l}\text { ATCC } \\
44774\end{array}$ \\
\hline DBY747 $\Delta m r s 2$ & $\begin{array}{l}\text { MATa leu2-3 leu2-112 his3-1 ura3-52 trp1-289 } \\
\text { mrs2::HIS3 }\end{array}$ & rho $^{+}$mit $^{+}$ & 31 \\
\hline DBY747 $\Delta$ grII & MATa leu2-3 leu2-112 his3-1 ura3-52 trp1-289 & $\begin{array}{l}\text { rho }{ }^{+} \text {mit }^{+} \Delta b I 1,2,3 \\
\Delta a I 1,2,5 a-c\end{array}$ & 31 \\
\hline DBY747 $\Delta$ grII $\Delta m r s 2$ (GW7/gd2-21.2/grII) & $\begin{array}{l}\text { MATa leu2-3 leu2-112 his3-1 ura3-52 trp1-289 } \\
\text { mrs2::HIS3 }\end{array}$ & $\begin{array}{l}\text { rho }{ }^{+} \text {mit }^{+} \Delta b I 1,2,3 \\
\Delta a I 1,2,5 a-c\end{array}$ & 31 \\
\hline DBY747 $\Delta$ grI $\Delta$ grII & MATa leu2-3 leu2-112 his3-1 ura3-52 trp1-289 & $\mathrm{rho}^{+}$mit $^{+} \Delta \Sigma b I, \Delta \Sigma a I, \Delta \omega$ & 31 \\
\hline DBY747 $\Delta \operatorname{grI} \Delta \operatorname{grII} \Delta m r s 2(G W 7 / g d 2-21.2 / w o)$ & $\begin{array}{l}\text { MATa leu2-3 leu2-112 his3-1 ura3-52 trp1-289 } \\
\text { mrs2::HIS3 }\end{array}$ & $\mathrm{rho}^{+} \mathrm{mit}^{+} \Delta \Sigma b I, \Delta \Sigma a I, \Delta \omega$ & 31 \\
\hline L40ccua & $\begin{array}{l}\text { MATa his3 } 200 \text { trp1-901 leu2-3,112 LYS2:: } \\
\text { (lexAop)4- HIS3 ura3::(lexAop)8-lacZ ADE2:: } \\
\text { (lexAop)8-URA3 GAL4 gal80 can1 cyh2 }\end{array}$ & & $\begin{array}{l}\text { E. E. } \\
\text { Wanker }\end{array}$ \\
\hline
\end{tabular}

times with PBS containing 3\% BSA. Samples were heated in SDS-sample buffer for five minutes at $95^{\circ} \mathrm{C}$ to elute bound protein from beads, and loaded onto a $12.5 \%$ SDS polyacrylamide gel. Subsequently, proteins were transferred onto nitrocellulose membrane (Schleicher and Schuell), and the membrane was blocked in 5\% milk solution. Afterwards, the membranes were incubated with antibodies directed against FLAG-tag (1:5000, Sigma) or HA-tag (1:5000, Sigma) for one hour, washed in PBS and incubated with peroxidase-conjugated antirabbit IgG $(1: 5000$, Sigma) or with peroxidaseconjugated anti-mouse IgG (1:5000, Sigma), respectively. Proteins were visualized by treating membranes with "westernlightning" luminol reagent (Perkin-Elmer).

\section{Confocal microscopy}

COS-1 cells were plated onto a glass slide and fixed after 18-24 hours with $4 \%$ paraformaldehyde for 15 minutes at RT. For induction of stress granules, cells were incubated for two hours at $42{ }^{\circ} \mathrm{C}$ before fixation. Afterwards, cells were incubated with PBS containing $0.05 \%$ Triton X-100 for 15 minutes and blocked in PBS supplemented with $3 \%$ BSA for 30 minutes. Subsequently, cells were incubated for double-staining with primary antibodies directed against PABP $(1: 400$, Abcam $)$ and ATX2 $\left(1: 300\right.$, named SCA2B by Huynh et al. $\left.{ }^{12}\right)$ for one hour at RT. After incubation with the respective secondary antibody conjugated to Cy3 or FITC, respectively, ( $1: 400$, Dianova), cells were washed with PBS and nuclei were stained with 4',6-diamidine-2-phenylindoledihydrochloride (DAPI, Sigma). An anti-fade mounting medium $(1 \mathrm{mg} / \mathrm{ml}$ p-phenylendiamine, $90 \%$ glycerol in PBS) was used for taking the micrographs. Confocal microscopy was performed with an LSM510 fluorescence microscope (Zeiss).

\section{In silico analysis}

We obtained protein sequences from the NCBI and UniProt databases, and retrieved domain architectures from the NCBI conserved domain (CD) database. ${ }^{78,79} \mathrm{We}$ searched sequence databases for homologous proteins of $\mathrm{ATX} 2, \mathrm{~A} 2 \mathrm{BP} 1$ and $\mathrm{Pbp} 1$ interaction partners using the (PSI-)BLAST and FASTA suite of programs with standard parameters. ${ }^{80,81}$ The multiple sequence alignment of A2BP1 and FOX-1 homologs (Supplementary Figure B) was computed by T-COFFEE ${ }^{82}$ and illustrated by the web tool ESPript. ${ }^{83}$ We used the online servers PROFsec,
PSIPRED and SAM-T99 to predict the secondary structures of A2BP1 and FOX-1 and annotated the multiple sequence alignment with the consensus formed as described by Albrecht et al. ${ }^{84}$ The GRID resource provided the annotation of yeast protein interaction data assembled from different sources for S. cerevisiae and allowed us to visualize it using the Osprey software. ${ }^{85}$

\section{Acknowledgements}

We are grateful to R. J. Schweyen and G. Wiesenberger (University of Vienna, Austria), E. E. Wanker (Max Delbrueck Center for Molecular Medicine, Berlin, Germany), for providing yeast strains and plasmids, to S. M. Pulst (Cedars-Sinai Medical Center, UCLA School of Medicine, Los Angeles, USA) for providing the SCA2B antibody, and to G. Auburger (Johann Wolfgang Goethe University, Frankfurt, Germany) for providing plasmid pcDNA1.1-Sca2Q22. Part of the research has been supported by the Max Planck Society and by the German Research Foundation (DFG) under contract number LE 491/14-1.

\section{Supplementary Data}

Supplementary data associated with this article can be found, in the online version, at doi:10.1016/j.jmb.2004.11.024

\section{References}

1. Pulst, S. M., Nechiporuk, A., Nechiporuk, T., Gispert, S., Chen, X. N., Lopes-Cendes, I. et al. (1996). Moderate expansion of a normally biallelic trinucleotide repeat in spinocerebellar ataxia type 2 . Nature Genet. 14, 269-276.

2. Sanpei, K., Takano, H., Igarashi, S., Sato, T., Oyake, M., Sasaki, H. et al. (1996). Identification of the 
spinocerebellar ataxia type 2 gene using a direct identification of repeat expansion and cloning technique, DIRECT. Nature Genet. 14, 277-284.

3. Imbert, G., Saudou, F., Yvert, G., Devys, D., Trottier, Y., Garnier, J. M. et al. (1996). Cloning of the gene for spinocerebellar ataxia 2 reveals a locus with high sensitivity to expanded CAG/glutamine repeats. Nature Genet. 14, 285-291.

4. Cummings, C. J. \& Zoghbi, H. Y. (2000). Trinucleotide repeats: mechanisms and pathophysiology. Annu. Rev. Genomics Hum. Genet. 1, 281-328.

5. Reddy, P. S. \& Housman, D. E. (1997). The complex pathology of trinucleotide repeats. Curr. Opin. Cell Biol. 9, 364-372.

6. Margolis, R. L. (2002). The spinocerebellar ataxias: order emerges from chaos. Curr. Neurol. Neurosci. Rep. 2, 447-456.

7. Stevanin, G., Dürr, A. \& Brice, A. (2002). Spinocerebellar ataxias caused by polyglutamine expansions. Advan. Expt. Med. Biol. 516, 47-77.

8. Bates, G. (2003). Huntingtin aggregation and toxicity in Huntington's disease. Lancet, 361, 1642-1644.

9. Harjes, P. \& Wanker, E. E. (2003). The hunt for huntingtin function: interaction partners tell many different stories. Trends Biochem. Sci. 28, 425-433.

10. Michalik, A. \& Van Broeckhoven, C. (2003). Pathogenesis of polyglutamine disorders: aggregation revisited. Hum. Mol. Genet. 12, R173-R186.

11. Huynh, D. P., Del Bigio, M. R., Ho, D. H. \& Pulst, S. M. (1999). Expression of ataxin-2 in brains from normal individuals and patients with Alzheimer's disease and spinocerebellar ataxia 2. Ann. Neurol. 45, 232-241.

12. Huynh, D. P., Figueroa, K., Hoang, N. \& Pulst, S. M. (2000). Nuclear localization or inclusion body formation of ataxin-2 are not necessary for SCA2 pathogenesis in mouse or human. Nature Genet. 26, $44-50$.

13. Huynh, D. P., Yang, H. T., Vakharia, H., Nguyen, D. \& Pulst, S. M. (2003). Expansion of the polyQ repeat in ataxin-2 alters its Golgi localization, disrupts the Golgi complex and causes cell death. Hum. Mol. Genet. 12, 1485-1496.

14. Wiedemeyer, R., Westermann, F., Wittke, I., Nowock, J. \& Schwab, M. (2003). Ataxin-2 promotes apoptosis of human neuroblastoma cells. Oncogene, 22, 401-411.

15. Meunier, C., Bordereaux, D., Porteu, F., Gisselbrecht, S., Chretien, S. \& Courtois, G. (2002). Cloning and characterization of a family of proteins associated with Mpl. J. Biol. Chem. 277, 9139-9147.

16. Shibata, H., Huynh, D. P. \& Pulst, S. M. (2000). A novel protein with RNA-binding motifs interacts with ataxin-2. Hum. Mol. Genet. 9, 1303-1313.

17. Jin, Y., Suzuki, H., Maegawa, S., Endo, H., Sugano, S., Hashimoto, K., Yasuda, K. \& Inoue, K. (2003). A vertebrate RNA-binding protein Fox-1 regulates tissue-specific splicing via the pentanucleotide GCAUG. EMBO J. 22, 905-912.

18. Neuwald, A. F. \& Koonin, E. V. (1998). Ataxin-2, global regulators of bacterial gene expression, and spliceosomal snRNP proteins share a conserved domain. J. Mol. Med. 76, 3-5.

19. Albrecht, M., Golatta, M., Wüllner, U. \& Lengauer, T. (2004). Structural and functional analysis of ataxin-2 and ataxin-3. Eur. J. Biochem. 271, 3155-3170.

20. He, W. \& Parker, R. (2000). Functions of Lsm proteins in mRNA degradation and splicing. Curr. Opin. Cell Biol. 12, 346-350.

21. Kiehl, T. R., Shibata, H. \& Pulst, S. M. (2000). The ortholog of human ataxin-2 is essential for early embryonic patterning in C. elegans. J. Mol. Neurosci. 15, 231-241.

22. Ciosk, R., DePalma, M. \& Priess, J. R. (2004). ATX-2, the C.elegans ortholog of ataxin 2, functions in translational regulation in the germline. Development, 131, 4831-4841.

23. Satterfield, T. F., Jackson, S. M. \& Pallanck, L. J. (2002). A Drosophila homolog of the polyglutamine disease gene SCA2 is a dosage-sensitive regulator of actin filament formation. Genetics, 162, 1687-1702.

24. Waldherr, M., Ragnini, A., Jank, B., Teply, R., Wiesenberger, G. \& Schweyen, R. J. (1993). A multitude of suppressors of group II intron-splicing defects in yeast. Curr. Genet. 24, 301-306.

25. Mangus, D. A., Amrani, N. \& Jacobson, A. (1998). Pbp1p, a factor interacting with Saccharomyces cerevisiae poly(A)-binding protein, regulates polyadenylation. Mol. Cell. Biol. 18, 7383-7396.

26. Mangus, D. A., Evans, M. C. \& Jacobson, A. (2003). Poly(A)-binding proteins: multifunctional scaffolds for the post-transcriptional control of gene expression. Genome Biol. 4, 223.1-223.14.

27. Mangus, D. A., Smith, M. M., McSweeney, J. M. \& Jacobson, A. (2004). Identification of factors regulating poly(A) tail synthesis and maturation. Mol. Cell. Biol. 24, 4196-4206.

28. Mangus, D. A., Evans, M. C., Agrin, N. S., Smith, M., Gongidi, P. \& Jacobson, A. (2004). Positive and negative regulation of poly(A) nuclease. Mol. Cell. Biol. 24, 5521-5533.

29. Tadauchi, T., Inada, T., Matsumoto, K. \& Irie, K. (2004). Posttranscriptional regulation of HO expression by the Mkt1-Pbp1 complex. Mol. Cell. Biol. 24, 3670-3681.

30. Kozlov, G., Trempe, J. F., Khaleghpour, K., Kahvejian, A., Ekiel, I. \& Gehring, K. (2001). Structure and function of the C-terminal PABC domain of human poly(A)-binding protein. Proc. Natl Acad. Sci. USA, 98, 4409-4413.

31. Albrecht, M. \& Lengauer, T. (2004). Novel Sm-like proteins with long C-terminal tails and associated methyltransferases. FEBS Letters, 569, 18-26.

32. Wiesenberger, G., Waldherr, M. \& Schweyen, R. J. (1992). The nuclear gene MRS2 is essential for the excision of group II introns from yeast mitochondrial transcripts in vivo. J. Biol. Chem. 267, 6963-6969.

33. Kolisek, M., Zsurka, G., Samaj, J., Weghuber, J., Schweyen, R. J. \& Schweigel, M. (2003). Mrs2p is an essential component of the major electrophoretic $\mathrm{Mg}^{2+}$ influx system in mitochondria. EMBO J. 22, 1235-1244.

34. Coller, J. M., Tucker, M., Sheth, U., Valencia-Sanchez, M. A. \& Parker, R. (2001). The DEAD box helicase, Dhh1p, functions in mRNA decapping and interacts with both the decapping and deadenylase complexes. RNA, 7, 1717-1727.

35. Maillet, L. \& Collart, M. A. (2002). Interaction between Not1p, a component of the Ccr4-not complex, a global regulator of transcription, and Dhh1p, a putative RNA helicase. J. Biol. Chem. 277, 2835-2842.

36. Tseng-Rogenski, S. S., Chong, J. L., Thomas, C. B., Enomoto, S., Berman, J. \& Chang, T. H. (2003). Functional conservation of Dhh1p, a cytoplasmic DExD/H-box protein present in large complexes. Nucl. Acids Res. 31, 4995-5002.

37. Nakielny, S. \& Dreyfuss, G. (1997). Nuclear export of proteins and RNAs. Curr. Opin. Cell Biol. 9, 420-429. 
38. Moroianu, J. (1998). Distinct nuclear import and export pathways mediated by members of the karyopherin beta family. J. Cell. Biochem. 70, 231-239.

39. Teixeira, M. T., Siniossoglou, S., Podtelejnikov, S., Bénichou, J. C., Mann, M., Dujon, B. et al. (1997). Two functionally distinct domains generated by in vivo cleavage of Nup145p: a novel biogenesis pathway for nucleoporins. EMBO J. 16, 5086-5097.

40. Floer, M. \& Blobel, G. (1999). Putative reaction intermediates in Crm1-mediated nuclear protein export. J. Biol. Chem. 274, 16279-16286.

41. Arselin, G., Vaillier, J., Graves, P. V. \& Velours, J. (1996). ATP synthase of yeast mitochondria. Isolation of the subunit $\mathrm{h}$ and disruption of the ATP14 gene. J. Biol. Chem. 271, 20284-20290.

42. Gerber, A. P. \& Keller, W. (1999). An adenosine deaminase that generates inosine at the wobble position of tRNAs. Science, 286, 1146-1149.

43. Bass, B. L. (2002). RNA editing by adenosine deaminases that act on RNA. Annu. Rev. Biochem. 71, 817-846.

44. Poulsen, H., Nilsson, J., Damgaard, C. K., Egebjerg, J. \& Kjems, J. (2001). CRM1 mediates the export of ADAR1 through a nuclear export signal within the Z-DNA binding domain. Mol. Cell. Biol. 21, 7862-7871.

45. Russnak, R., Pereira, S. \& Platt, T. (1996). RNA binding analysis of yeast REF2 and its two-hybrid interaction with a new gene product, FIR1. Gene Expr. 6, 241-258.

46. del Olmo, M., Mizrahi, N., Gross, S. \& Moore, C. L. (1997). The Uba2 and Ufd1 proteins of Saccharomyces cerevisiae interact with poly(A) polymerase and affect the polyadenylation activity of cell extracts. Mol. Gen. Genet. 255, 209-218.

47. Breitkreutz, A., Boucher, L., Breitkreutz, B. J., Sultan, M., Jurisica, I. \& Tyers, M. (2003). Phenotypic and transcriptional plasticity directed by a yeast mitogenactivated protein kinase network. Genetics, 165, 997-1015.

48. Gelli, A. (2002). Rst1 and Rst2 are required for the a/ $\alpha$ diploid cell type in yeast. Mol. Microbiol. 46, 845-854.

49. Kasten, M. M. \& Stillman, D. J. (1997). Identification of the Saccharomyces cerevisiae genes STB1-STB5 encoding Sin3p binding proteins. Mol. Gen. Genet. 256, 376-386.

50. Takagi, T., Cho, E. J., Janoo, R. T., Polodny, V., Takase, Y., Keogh, M. C. et al. (2002). Divergent subunit interactions among fungal mRNA 5'-capping machineries. Eukaryot. Cell, 1, 448-457.

51. Parisi, M. \& Lin, H. (2000). Translational repression: a duet of Nanos and Pumilio. Curr. Biol. 10, R81-R83.

52. Olivas, W. \& Parker, R. (2000). The Puf3 protein is a transcript-specific regulator of mRNA degradation in yeast. EMBO J. 19, 6602-6611.

53. Wickens, M. \& Goldstrohm, A. (2003). Molecular biology. A place to die, a place to sleep. Science, $\mathbf{3 0 0 ,}$ 753-755.

54. Albrecht, M. \& Lengauer, T. (2004). Survey on the PABC recognition motif PAM2. Biochem. Biophys. Res. Commun. 316, 129-138.

55. Kedersha, N. \& Anderson, P. (2002). Stress granules: sites of mRNA triage that regulate mRNA stability and translatability. Biochem. Soc. Trans. 30, 963-969.

56. Tarun, S. Z., Jr \& Sachs, A. B. (1996). Association of the yeast poly $(\mathrm{A})$ tail binding protein with translation initiation factor eIF-4G. EMBO J. 15, 7168-7177.

57. Kedersha, N., Cho, M. R., Li, W., Yacono, P. W., Chen,
S., Gilks, N. et al. (2000). Dynamic shuttling of TIA-1 accompanies the recruitment of mRNA to mammalian stress granules. J. Cell Biol. 151, 1257-1268.

58. Kai, N., Mishina, M. \& Yagi, T. (1997). Molecular cloning of Fyn-associated molecules in the mouse central nervous system. J. Neurosci. Res. 48, 407-424.

59. Norris, J. D., Fan, D., Sherk, A. \& McDonnell, D. P. (2002). A negative coregulator for the human ER. Mol. Endocrinol. 16, 459-468.

60. Lieberman, A. P., Friedlich, D. L., Harmison, G., Howell, B. W., Jordan, C. L., Breedlove, S. M. \& Fischbeck, K. H. (2001). Androgens regulate the mammalian homologues of invertebrate sex determination genes tra-2 and fox-1. Biochem. Biophys. Res. Commun. 282, 499-506.

61. Hodgkin, J., Zellan, J. D. \& Albertson, D. G. (1994). Identification of a candidate primary sex determination locus, fox -1 , on the $X$ chromosome of Caenorhabditis elegans. Development, 120, 3681-3689.

62. Nicoll, M., Akerib, C. C. \& Meyer, B. J. (1997). $\mathrm{X}$-Chromosome-counting mechanisms that determine nematode sex. Nature, 388, 200-204.

63. Skipper, M., Milne, C. A. \& Hodgkin, J. (1999). Genetic and molecular analysis of fox-1, a numerator element involved in Caenorhabditis elegans primary sex determination. Genetics, 151, 617-631.

64. Meyer, B. J. (2000). Sex in the worm counting and compensating X-chromosome dose. Trends Genet. 16, 247-253.

65. Gomes, J. E., Encalada, S. E., Swan, K. A., Shelton, C. A., Carter, J. C. \& Bowerman, B. (2001). The maternal gene spn- 4 encodes a predicted RRM protein required for mitotic spindle orientation and cell fate patterning in early C.elegans embryos. Development, 128, 4301-4314.

66. Huang, N. N., Mootz, D. E., Walhout, A. J., Vidal, M. \& Hunter, C. P. (2002). MEX-3 interacting proteins link cell polarity to asymmetric gene expression in Caenorhabditis elegans. Development, 129, 747-759.

67. Labbé, J. C. \& Goldstein, B. (2002). Embryonic development: a new SPN on cell fate specification. Curr. Biol. 12, R396-R398.

68. Tsuboi, D., Qadota, H., Kasuya, K., Amano, M. \& Kaibuchi, K. (2002). Isolation of the interacting molecules with GEX-3 by a novel functional screening. Biochem. Biophys. Res. Commun. 292, 697-701.

69. Ogura, K., Kishimoto, N., Mitani, S., Gengyo-Ando, K. \& Kohara, Y. (2003). Translational control of maternal glp-1 mRNA by POS-1 and its interacting protein SPN-4 in Caenorhabditis elegans. Development, 130, 2495-2503.

70. Soto, M. C., Qadota, H., Kasuya, K., Inoue, M., Tsuboi, D., Mello, C. C. \& Kaibuchi, K. (2002). The GEX-2 and GEX-3 proteins are required for tissue morphogenesis and cell migrations in C. elegans. Genes Dev. 16, 620-632.

71. Blagg, S. L. \& Insall, R. H. (2004). Solving the WAVE function. Nature Cell Biol. 6, 279-281.

72. Chen, Y. W., Allen, M. D., Veprintsev, D. B., Lowe, J. \& Bycroft, M. (2004). The structure of the AXH domain of spinocerebellar ataxin-1. J. Biol. Chem. 279, 3758-3765.

73. de Chiara, C., Giannini, C., Adinolfi, S., de Boer, J., Guida, S., Ramos, A. et al. (2003). The AXH module: an independently folded domain common to ataxin-1 and HBP1. FEBS Letters, 551, 107-112.

74. Yue, S., Serra, H. G., Zoghbi, H. Y. \& Orr, H. T. (2001). The spinocerebellar ataxia type 1 protein, ataxin-1, 
has RNA-binding activity that is inversely affected by the length of its polyglutamine tract. Hum. Mol. Genet. 10, 25-30.

75. Fernandez-Funez, P., Nino-Rosales, M. L., de Gouyon, B., She, W. C., Luchak, J. M., Martinez, P. et al. (2000). Identification of genes that modify ataxin-1-induced neurodegeneration. Nature, 408, 101-106.

76. Wanker, E. E., Rovira, C., Scherzinger, E., Hasenbank, R., Walter, S., Tait, D. et al. (1997). HIP-I: a huntingtin interacting protein isolated by the yeast two-hybrid system. Hum. Mol. Genet. 6, 487-495.

77. Schiestl, R. H. \& Gietz, R. D. (1989). High efficiency transformation of intact yeast cells using single stranded nucleic acids as a carrier. Curr. Genet. 16, 339-346.

78. Wheeler, D. L., Church, D. M., Edgar, R., Federhen, S., Helmberg, W., Madden, T. L. et al. (2004). Database resources of the National Center for Biotechnology Information: update. Nucl. Acids Res. 32, D35-D40.

79. Apweiler, R., Bairoch, A., Wu, C. H., Barker, W. C., Boeckmann, B., Ferro, S. et al. (2004). UniProt: the Universal Protein knowledgebase. Nucl. Acids Res. 32, D115-D119.
80. Altschul, S. F., Madden, T. L., Schaffer, A. A., Zhang, J., Zhang, Z., Miller, W. \& Lipman, D. J. (1997). Gapped BLAST and PSI-BLAST: a new generation of protein database search programs. Nucl. Acids Res. 25, 3389-3402.

81. Pearson, W. R. (2000). Flexible sequence similarity searching with the FASTA3 program package. Methods Mol. Biol. 132, 185-219.

82. Poirot, O., O'Toole, E. \& Notredame, C. (2003) Tcoffee@igs: a web server for computing, evaluating and combining multiple sequence alignments. Nucl. Acids Res. 31, 3503-3506.

83. Gouet, P., Robert, X. \& Courcelle, E. (2003). ESPript/ ENDscript: extracting and rendering sequence and 3D information from atomic structures of proteins. Nucl. Acids Res. 31, 3320-3323.

84. Albrecht, M., Tosatto, S. C., Lengauer, T. \& Valle, G. (2003). Simple consensus procedures are effective and sufficient in secondary structure prediction. Protein Eng. 16, 459-462.

85. Breitkreutz, B. J., Stark, C. \& Tyers, M. (2003). Osprey: a network visualization system. Genome Biol. 4, R22.1-R22.4.

Edited by F. E. Cohen

(Received 5 October 2004; accepted 5 November 2004) 